\title{
A Pilot Clinical Trial of Repetitive Transcranial Magnetic Stimulation in Mild Cognitive Impairment
}

\author{
Sara Esmaeilia, b, c, Mohammad Hossein Abbasia, b, c, Ensieh Malekdar ${ }^{\mathrm{a}}$, \\ Mohammad Taghi Joghataeic, Masoud Mehrpoura, d
}

\begin{abstract}
Background: Mild cognitive impairment (MCI) is defined as a progressive memory dysfunction. There are controversies with regards to whether repetitive transcranial magnetic stimulation (rTMS) could improve the condition.

Methods: In a randomized, self-control, crossover clinical trial, effect of rTMS on cognitive performance in patients with MCI was assessed. Patients were randomized into two study groups (A and $\mathrm{B}$ ) and received both rTMS procedure and sham therapy in sequence, with each lasting for 8 weeks. Montreal cognitive assessment (MoCA) test was performed as a cognition battery at baseline and 1 week after each 8-week period of interventions.

Results: Sixteen patients were enrolled in the study. Baseline measures of MoCA were statistically equal between two groups ( $\mathrm{P}$ value $=0.10$ ). Mean MoCA score significantly increased in group $A$ at nine-week follow-up compared to both group $\mathrm{B}(\mathrm{P}$ value $<0.001)$ and its baseline $(\mathrm{P}$ value $=0.01)$. However, at 18 -week follow-up, mean MoCA scores were increased in both groups compared to their baseline (both $\mathrm{P}$ values $<0.001$ ) with no significant differences between study groups $(\mathrm{P}$ value $=0.87)$. No adverse effects were reported.
\end{abstract}

Conclusions: The rTMS is suggested as an effective and safe therapeutic option for cognitive improvement in patients with MCI.

Keywords: Mild cognitive impairment; Repetitive transcranial magnetic stimulation; rTMS; MoCA

Manuscript submitted July 31, 2020, accepted August 6, 2020

Published online August 15, 2020

${ }^{a}$ Neurology Department, Firoozgar Hospital, Iran University of Medical Sciences, Tehran, Iran

${ }^{\mathrm{b} S t u d e n t ~ R e s e a r c h ~ C o m m i t t e e, ~ I r a n ~ U n i v e r s i t y ~ o f ~ M e d i c a l ~ S c i e n c e s, ~ T e h r a n, ~ I r a n ~}$ ${ }^{\mathrm{c}}$ Cellular and Molecular Research Center, Iran University of Medical Sciences, Tehran, Iran

${ }^{\mathrm{d} C o r r e s p o n d i n g ~ A u t h o r: ~ M a s o u d ~ M e h r p o u r, ~ M e d i c a l ~ F a c u l t y, ~ I r a n ~ U n i v e r s i t y ~}$ of Medical Sciences (IUMS), Hemmat Highway, Tehran Province, Tehran, Iran. Email: dr.masoudmehrpour@gmail.com

doi: https://doi.org/10.14740/jnr622

\section{Introduction}

Mild cognitive impairment (MCI) is defined as a progressive memory dysfunction and a transitional state between normal cognitive performance and dementia [1,2]. MCI interrupts with daily activities and it might convert to dementia with $10 \%$ risk per year [1,2]. Previous studies have shown unsatisfying results of medications in terms of clinical improvement [1-3].

Applying repetitive transcranial magnetic stimulation (rTMS) over the prefrontal cortex is a non-invasive method which has shown desirable therapeutic effects in a wide range of different neurologic disorders including major depression, neuropathic pain, post-stroke neurorehabilitation, as well as multiple sclerosis (MS), amyotrophic lateral sclerosis (ALS), stroke and migraine [4-9]; yet its role in enhancing cognition remains controversial. The role of prefrontal cortex (PFC) in cognition and memory retrieval has previously been highlighted as the best target of stimulation in order to regain its function with subsequent improvement in cognitive and memory performance [10]. rTMS affects brain cortical neurons by modulating the excitability and monoamine release which finally expedites plasticity in cortical gray matter at memory-associated areas [11].

The aim of this study is to evaluate rTMS applicability over the left dorsolateral prefrontal cortex (DLPFC) area as a therapeutic option in MCI by repetitive cognition assessments in a crossover self-control clinical trial.

\section{Materials and Methods}

The study is a crossover self-control randomized clinical trial. Study design has been shown in Figure 1.

\section{Enrolment}

Study population consisted of a consecutive sampling of 16

\section{Key Points}

- rTMS applied over the left DLPFC improved MoCA scores in patients with MCI.

- The effect of rTMS lasted up to 18-week follow-up.

- rTMS was safe with no adverse effects. 


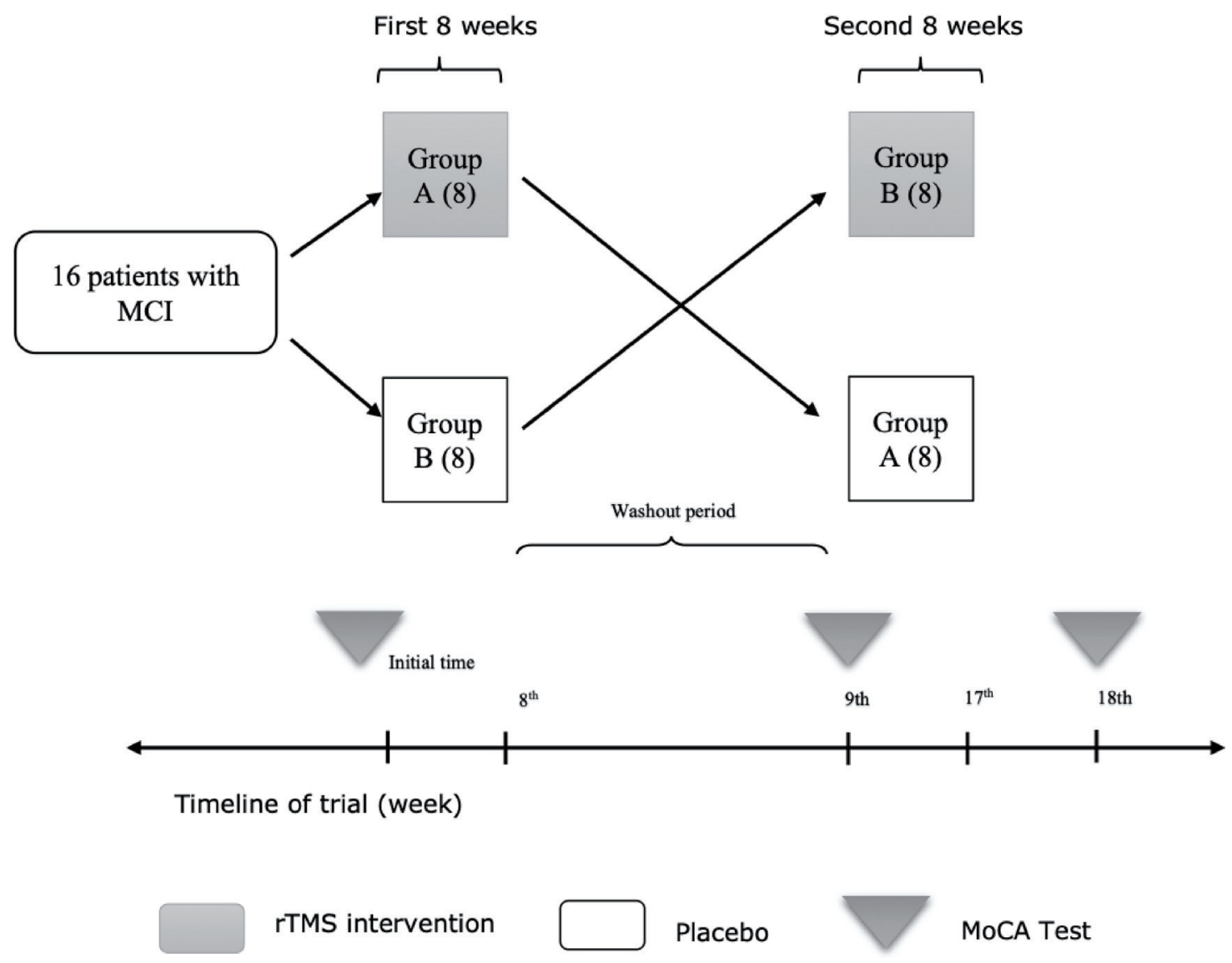

Figure 1. Method describing flowchart. $\mathrm{MCl}$ : mild cognitive impairment; rTMS: repetitive transcranial magnetic stimulation; MoCA: Montreal cognitive assessment.

patients referred to neurology clinics of two central hospitals named Firoozgar and Rasoul-e-Akram hospitals affiliated to Iran University of Medical Sciences (IUMS), Tehran, Iran. Inclusion criteria included patients aged between 20 - 70 years old and baseline Montreal cognitive assessment (MoCA) score below 26 as the predefined cut-off point for MCI [12]. Exclusion criteria comprised patients with cardiac pacemakers, psychiatric disorders, and patients with secondary causes of cognitive decline including prior history of brain injury accompanied with loss of consciousness, medication history of benzodiazepines or alcohol, or theophylline, serum B12 deficiency, hypothyroidism, and structural lesions of the brain.

\section{Treatment allocation}

Patients were equally allocated into two groups "A" and "B" by block randomization. Block randomization contained eight binary blocks of A and B for random allocation of samples in two study groups. Group "A" was treated by rTMS in the first eight sessions while group "B" received sham stimulation. One week after the end of first eight sessions, interventions were switched between groups in a crossover manner; group "A" received sham stimulation while group
"B" was treated by rTMS for another eight sessions. Patients were unaware of the allocation sequence or the type of the intervention, though the investigators were not blinded to that. Deterioration of patient's clinical status was considered as the indication of exclusion from this study according to research ethics protocol.

\section{Intervention}

We performed the intervention based on a method which has previously been described by Sole-Padulles et al [13]. rTMS was performed by a device named Medtronic, manufactured in Denmark. A figure-of-eight coil was positioned over the cortical area, and small parts of the brain were stimulated by a magnetic generator. The magnetic flux was applied over a certain area called F3 point at DLPFC on the cerebral cortex of left hemisphere.

Stimulation was applied on wake patients without anesthesia who were fixed on a comfortable chair for duration of $30 \mathrm{~min}$. The duration of each pulse was $10 \mathrm{~s}$, and the pulse repetition interval (PRI) which is known as the time interval between pulses intervals was $20 \mathrm{~s}$. Thus, each patient received a total of 60 pulses in $30 \mathrm{~min}$. Patients in this study were treated for 16 sessions, two sessions per week. Each group changed its intervention type one 
Table 1. Descriptive Statistics of Cognition Assessment and Inter Group Analysis of MoCA Scores

\begin{tabular}{lllll}
\hline Cognition parameter assessments & Group category $(\mathbf{n})$ & Mean & SD & P value of comparison between groups (CI) \\
\hline MoCA: baseline & A (8) & 25.18 & 0.90 & $0.10(-0.09-1.88)$ \\
& B (8) & 24.29 & 1.16 & \\
MoCA: 1 week after the 8th session & A (8) & 26.02 & 0.77 & $<0.001(0.7-2.68)$ \\
& B (8) & 24.33 & 0.68 & \\
MoCA: 1 week after the 16th session & A (8) & 26.77 & 0.68 & $0.87(-0.61-1.37)$ \\
& B (8) & 26.39 & 0.87 & \\
\hline
\end{tabular}

SD: standard deviation; $\mathrm{Cl}$ : confidence interval; MoCA: Montreal cognitive assessment.

week after the first eight sessions. To avoid placebo bias, sham intervention was applied over the same area on the scalp by the same device and the same clinical setting. However, the wires of magnetic generator were disconnected from the device.

\section{Follow-up}

MoCA test approved by the American Academy of Neurology (AAN) as a valid tool in assessment of cognitive function was selected in this study. MoCA scores were measured at baseline, 1 week after the eighth session (nine-week follow-up) and 1 week after the 16th session (18-week follow-up) of interventions in both groups.

\section{Analysis}

Sample size of 16 was planned for this study by assumption of normal distribution and homogeneity of variances to compare two independent groups. Alpha error of 0.05 was determined with power of $80 \%$ and precision of measures equal to 2 score $(\Delta=$ $\left.\mu_{1}-\mu_{2}\right)$. Predefined mean and variance of MoCA among MCI patients $(25.1 \pm 1.94)$ were considered. In order to compare MoCA score, analysis of variance (ANOVA) with repeated measures was performed within and inter groups prior to interventions, at nineweek follow-up and at 18-week follow-up. Statistical data were analyzed using SPSS version 23.

\section{Ethics}

This study is an internationally registered clinical trial with IRCT registration number: IRCT2017011028199N2. The study was performed according to Helsinki ethics protocols and was approved by Ethics Committee of Iran University of Medical
Sciences (Code IR.IUMS.REC 1394.9211158008)

\section{Results}

Sixteen patients with MCI were recruited to the study with eight patients in each group. There were totally five females and 11 males in the study with average age of 53.12 years. Descriptive statistics of MoCA scores of three repeated sessions are shown in (Table 1). Mean MoCA scores were not statistically different at baseline between study groups $(\mathrm{P}$ value $=0.10)$.

\section{Analysis within the groups}

Compared to baseline, mean MoCA score improved significantly at nine-week follow-up in group A compared to baseline, while group B (sham group) showed no improvement of mean MoCA score at nine-week follow-up compared to its baseline. Assessments at 18-week follow-up showed significant improvements in mean MoCA scores of both groups compared to their baseline measures (Table 2).

\section{Inter groups analysis}

Comparing outcomes between groups, group A had statistically higher mean MoCA score at nine-week follow-up compared to group B ( $\mathrm{P}$ value $<0.001)$ while data showed no statistically significant difference between two groups at 18-week followup $(\mathrm{P}$ value $=0.87)($ Table 1$)$.

\section{Adverse effects}

No adverse effects of rTMS were reported during the study.

Table 2. Within Group Analysis of MoCA Scores

\begin{tabular}{llll}
\hline Cognition parameter assessments & Group category $(\mathbf{n})$ & P value (effect compared to before study measures) & Confidence interval \\
\hline MoCA: 1 week after the 8th session & A $(8)$ & 0.017 & $(0.16-1.52)$ \\
& B (8) & 1.00 & $(-0.94-1.03)$ \\
MoCA: 1 week after the 16th session & A (8) & $<0.001$ & $(0.60-2.58)$ \\
& B (8) & $<0.001$ & $(1.11-3.09)$ \\
\hline
\end{tabular}




\section{Discussion}

The present study has found favorable effects of rTMS applied over the left DLPFC after multiple sessions on cognitive performance in patients with MCI.

Two groups were similar in terms of cognitive function at baseline. On nine-week follow-up, cognition was significantly improved in group A compared to both its baseline measures and the simultaneous cognitive performance scores in sham group. However, intragroup analysis showed no statistically significant differences between two groups' MoCA scores on 18-week follow-up. These findings indicate that rTMS was the reason of cognition improvement in each group compared to sham therapy. These results also revealed that rTMS effects could last up to 18 weeks. However, studies with longer follow-up should be performed in order to assess longer-term effects of rTMS. Our results are similar to previous studies which showed that high frequency rTMS over the left parietal cortex had satisfactory effects on clinical status of MCI [1417].

DLPFC region is well known for its role in verbal and nonverbal memory retrieval along with cognitive process [18-23]. It has been suggested that stimulation of left DLPFC improves cognition and memory simultaneously [24]. Stimulation of DLPC area by rTMS improves working memory, which has been shown to last for up to 24 weeks [24]. As well as the impact on cognition, it has been argued that rTMS could improve the quality of life indirectly by improving performance of everyday activities in MCI patients [25].

The mechanism by which rTMS affects the cortex is obscure. It is believed that rTMS has direct modulating effects on neuronal activity and plasticity especially on excitatory synapses [11, 26-28]. A previous study showed that stimulation of the left DLPFC increases the metabolic activity in this area which in turn improves cognition in older people with memory disorders [13].

Our patients reported no adverse effects according to rTMS. This technique has been demonstrated as a safe method with minimal serious adverse events claimed by previous clinical trials in the past two decades since the first clinical application [29].

One of the limitations of our study was that our patients were not followed up for a long time to investigate long-term effect of rTMS on MoCA scores. Studies comparing rTMS with medications are recommended.

\section{Conclusions}

The results of this study show that rTMS possibly plays an improving role in patients with MCI.

\section{Acknowledgments}

This study has been funded by the Iran University of Medical Science which is greatly appreciated. Authors would like to thank Maryam Daneshgar for her kind help in English edition and Dr. Peyman Shirani for his meaningful support.

\section{Financial Disclosure}

This study has been funded by the Iran University of Medical Science (IUMS).

\section{Conflict of Interest}

The authors certify that they have no affiliations with or involvement in any organization or entity with any financial or non-financial in the subject matter or materials discussed in this manuscript. All authors report no disclosures.

\section{Informed Consent}

Signed informed consents were obtained.

\section{Author Contributions}

Sara Esmaeili: conception, execution, writing of the first draft, review and critique; Mohammad Hossein Abbasi: conception, execution, statistical analysis, and writing of the first draft. Ensieh Malekdar: conception, execution, and writing of the first draft. Mohammad Taghi Joghataei: conception, organization, review and critique. Masoud Mehrpour: conception, organization, execution, review and critique.

\section{Data Availability}

The data supporting the findings of this study are available from the corresponding author upon reasonable request.

\section{References}

1. Petersen RC, Smith GE, Waring SC, Ivnik RJ, Tangalos EG, Kokmen E. Mild cognitive impairment: clinical characterization and outcome. Arch Neurol. 1999;56(3):303308.

2. Bischkopf J, Busse A, Angermeyer MC. Mild cognitive impairment - a review of prevalence, incidence and outcome according to current approaches. Acta Psychiatr Scand. 2002;106(6):403-414.

3. Strohle A, Schmidt DK, Schultz F, Fricke N, Staden T, Hellweg R, Priller J, et al. Drug and exercise treatment of Alzheimer disease and mild cognitive impairment: a systematic review and meta-analysis of effects on cognition in randomized controlled trials. Am J Geriatr Psychiatry. 2015;23(12):1234-1249.

4. Leon Ruiz M, Rodriguez Sarasa ML, Sanjuan Rodriguez L, Benito-Leon J, Garcia-Albea Ristol E, Arce Arce S. 
Current evidence on transcranial magnetic stimulation and its potential usefulness in post-stroke neurorehabilitation: Opening new doors to the treatment of cerebrovascular disease. Neurologia. 2018;33(7):459-472.

5. Iodice R, Manganelli F, Dubbioso R. The therapeutic use of non-invasive brain stimulation in multiple sclerosis - a review. Restor Neurol Neurosci. 2017;35(5):497-509.

6. Ng L, Khan F, Young CA, Galea M. Symptomatic treatments for amyotrophic lateral sclerosis/motor neuron disease. Cochrane Database Syst Rev. 2017;1:CD011776.

7. McClintock SM, Reti IM, Carpenter LL, McDonald WM, Dubin M, Taylor SF, Cook IA, et al. Consensus recommendations for the clinical application of repetitive Transcranial Magnetic Stimulation (rTMS) in the treatment of depression. J Clin Psychiatry. 2018;79(1).

8. Boldt I, Eriks-Hoogland I, Brinkhof MW, de Bie R, Joggi D, von Elm E. Non-pharmacological interventions for chronic pain in people with spinal cord injury. Cochrane Database Syst Rev. 2014;11(11):CD009177.

9. Schwedt TJ, Vargas B. Neurostimulation for treatment of migraine and cluster headache. Pain Med. 2015;16(9):1827-1834.

10. Brunoni AR, Vanderhasselt MA. Working memory improvement with non-invasive brain stimulation of the dorsolateral prefrontal cortex: a systematic review and meta-analysis. Brain Cogn. 2014;86:1-9.

11. Rossi S, Hallett M, Rossini PM, Pascual-Leone A, Safety of TMSCG. Safety, ethical considerations, and application guidelines for the use of transcranial magnetic stimulation in clinical practice and research. Clin Neurophysiol. 2009;120(12):2008-2039.

12. Dautzenberg G, Lijmer J, Beekman A. Diagnostic accuracy of the Montreal Cognitive Assessment (MoCA) for cognitive screening in old age psychiatry: Determining cutoff scores in clinical practice. Avoiding spectrum bias caused by healthy controls. Int J Geriatr Psychiatry. 2020;35(3):261-269.

13. Sole-Padulles C, Bartres-Faz D, Junque C, Clemente IC, Molinuevo JL, Bargallo N, Sanchez-Aldeguer J, et al. Repetitive transcranial magnetic stimulation effects on brain function and cognition among elders with memory dysfunction. A randomized sham-controlled study. Cereb Cortex. 2006;16(10):1487-1493.

14. Bai F, Zhang Z, Watson DR, Yu H, Shi Y, Yuan Y, Zang $\mathrm{Y}$, et al. Abnormal functional connectivity of hippocampus during episodic memory retrieval processing network in amnestic mild cognitive impairment. Biol Psychiatry. 2009;65(11):951-958.

15. Petersen RC, Stevens JC, Ganguli M, Tangalos EG, Cummings JL, DeKosky ST. Practice parameter: early detection of dementia: mild cognitive impairment (an evidence-based review). Report of the Quality Standards Subcommittee of the American Academy of Neurology. Neurology. 2001;56(9):1133-1142.

16. Wang L, Zang Y, He Y, Liang M, Zhang X, Tian L, Wu $\mathrm{T}$, et al. Changes in hippocampal connectivity in the early stages of Alzheimer's disease: evidence from resting state
fMRI. Neuroimage. 2006;31(2):496-504

17. Ready RE, Ott BR, Grace J, Cahn-Weiner DA. Apathy and executive dysfunction in mild cognitive impairment and Alzheimer disease. Am J Geriatr Psychiatry. 2003;11(2):222-228.

18. Turriziani P, Smirni D, Zappala G, Mangano GR, Oliveri M, Cipolotti L. Enhancing memory performance with rTMS in healthy subjects and individuals with mild cognitive impairment: the role of the right dorsolateral prefrontal cortex. Front Hum Neurosci. 2012;6:62.

19. Vijayakumari AA, Menon RN, Thomas B, Arun TM, Nandini M, Kesavadas C. Glutamatergic response to a low load working memory paradigm in the left dorsolateral prefrontal cortex in patients with mild cognitive impairment: a functional magnetic resonance spectroscopy study. Brain Imaging Behav. 2020;14(2):451-459.

20. Zhang X, Yu JT, Li J, Wang C, Tan L, Liu B, Jiang T. Bridging Integrator 1 (BIN1) genotype effects on working memory, hippocampal volume, and functional connectivity in young healthy individuals. Neuropsychopharmacology. 2015;40(7):1794-1803.

21. Gigi A, Babai R, Penker A, Hendler T, Korczyn AD. Prefrontal compensatory mechanism may enable normal semantic memory performance in mild cognitive impairment (MCI). J Neuroimaging. 2010;20(2):163-168.

22. Qin S, Hermans EJ, van Marle HJ, Luo J, Fernandez G. Acute psychological stress reduces working memoryrelated activity in the dorsolateral prefrontal cortex. Biol Psychiatry. 2009;66(1):25-32.

23. Li Y, Wang L, Jia M, Guo J, Wang H, Wang M. The effects of high-frequency rTMS over the left DLPFC on cognitive control in young healthy participants. PLoS One. 2017;12(6):e0179430.

24. Cotelli M, Calabria M, Manenti R, Rosini S, Maioli C, Zanetti O, Miniussi C. Brain stimulation improves associative memory in an individual with amnestic mild cognitive impairment. Neurocase. 2012;18(3):217-223.

25. Nardone R, Tezzon F, Holler Y, Golaszewski S, Trinka E, Brigo F. Transcranial magnetic stimulation (TMS)/repetitive TMS in mild cognitive impairment and Alzheimer's disease. Acta Neurol Scand. 2014;129(6):351-366.

26. Mano Y, Morita Y, Tamura R, Morimoto S, Takayanagi $\mathrm{T}$, Mayer RF. The site of action of magnetic stimulation of human motor cortex in a patient with motor neuron disease. J Electromyogr Kinesiol. 1993;3(4):245-250.

27. Mano Y, Nakamuro T, Ikoma K, Takayanagi T, Mayer RF. A clinicophysiologic study of central and peripheral motor conduction in hereditary demyelinating motor and sensory neuropathy. Electromyogr Clin Neurophysiol. 1993;33(2):101-107.

28. Sato A, Torii T, Nakahara Y, Iwahashi M, Itoh Y, Iramina $\mathrm{K}$. The impact of rTMS over the dorsolateral prefrontal cortex on cognitive processing. Conf Proc IEEE Eng Med Biol Soc. 2013;2013:1988-1991.

29. Dodick DW, Schembri CT, Helmuth M, Aurora SK. Transcranial magnetic stimulation for migraine: a safety review. Headache. 2010;50(7):1153-1163. 